CIFRAS DE HOY

Rev Chil Salud Pública 2018 , Vol 22(2): 188-196
NELIO BAZÁN Universidad Nacional de Villa Mercedes nelio.bazan@gmail.com

CLAUDIA VALENTI Ministerio de Salud, GCBA

FERNANDO A. LAIÑO

Fundación Instituto Superior de Ciencias de la Salud (CABA) y Universidad Nacional de Villa Mercedes (San Luis), 2016.

Articulo recibido el 26/10/2016 Aceptado el 12/09/2017

\section{MEDIPAQ 2. ACTIVIDAD FÍSICA, CONDUCTA SEDENTARIA Y CIRCUNFERENCIA DE CINTURA EN PERSONAL DE SALUD DEL AREA METROPOLITANA DE BUENOS AIRES}

\author{
MEDIPAQ2. Physical activity, Sedentary behavior, waist \\ CIRCUMFERENCE IN HEALTH PERSONNEL FROM THE METROPOLITAN \\ AREA OF BUENOS AIRES
}

\section{INTRODUCCIÓN}

La enfermedad cardiovascular es considerada la primera causa de morbilidad y mortalidad en la población adulta a nivel mundial. La lesión arterial aterosclerótica tiene como factores de riesgo a la obesidad, las dislipemias, la hipertensión arterial, el hábito tabáquico, la diabetes mellitus y la insuficiente actividad física. ${ }^{1}$ La modificación de estos factores de riesgo es una excelente estrategia para evitar o retardar la aparición de la noxa. ${ }^{2}$ La obesidad, o sea, el exceso de grasa corporal resultante del desequilibrio crónico entre la ingesta y el gasto energético, ${ }^{3}$ está asociada a la hipertensión arterial, la diabetes, las dislipemias y a algunos tipos de cáncer. ${ }^{4}$ La intervención nutricional y la realización de actividad física son las intervenciones imprescindibles para equilibrar el consumo con el gasto energético. La educación sanitaria debe colaborar ayudando a modificar hábitos y a adoptar costumbres saludables como realizar actividad física desde la infancia. Esto debiera facilitar que los adultos realicen actividad física en cantidad y calidad adecuadas a su edad y nivel de aptitud física. La práctica de ejercicio físico regular también influye positivamente en la adopción de otros comportamientos saludables relacionados con la alimentación adecuada o la disminución del consumo de alcohol y cese del hábito tabáquico. Pero aunque existe ya consenso en la población general que realizar actividad física es saludable, esto no siempre se traduce en un comportamiento consecuente.

Los médicos son una fuente creíble y respetada de información relacionada con la salud que puede proporcionar un asesoramiento preventivo y realizar seguimientos. También, tienen una obligación ética de prescribir un estilo de vida saludable como base del tratamiento de las enfermedades crónicas no transmisibles. Si esto ocurriese realmente, se convertirían en una importante intervención respecto a la prevención de enfermedades crónicas. Ellos tienen el potencial de aumentar los niveles de actividad física en la población con una adecuada prescripción de actividad física y ejercicio en sus pacientes como un modo de realizar prevención primaria y secundaria de muchas enfermedades como las crónicas no transmisibles: obesidad, diabetes y enfermedad cardiovascular, entre otras. ${ }^{5,9}$ Pero existen barreras para prescribir correctamente actividad física, que van desde la exigua formación sobre el tema en los estudios de grado, hasta la motivación insuficiente relacionada con escasos hábitos de actividad física personal. Las tasas de prescripción de ejercicio por parte de los médicos son bajas en los Estados Unidos. Solo el 34\% de los pacientes adultos lo recibe y esto sucede en la mayoría de los países. ${ }^{10}$ Se considera, por lo tanto, 
necesario incrementar la proporción de pacientes a los que se informe acerca de los comportamientos saludables como la actividad física. Los médicos que actúan en relación a lo que aconsejan (incluyendo actividad física) son más eficientes en esa recomendación y pueden lograr una mayor motivación de sus pacientes. Hay fuerte evidencia de que los médicos que son físicamente activos, favorecen la prescripción de actividad física de sus pacientes, en su práctica profesional. ${ }^{11}$ En ese sentido, resulta interesante estudiar la actividad física que realizan las personas involucradas en los servicios de salud (aquellos que deberían aconsejar sobre actividad física a sus pacientes). Así, el presente trabajo tuvo como objetivo estudiar el nivel de actividad física, la conducta sedentaria y el riesgo metabólico de personal de salud (médicos, enfermeros y personal técnico) del área metropolitana de la ciudad de Buenos Aires.

\section{MATERIAL Y MÉTODO}

Esta investigación es de tipo observacional y transversal, de nivel descriptivo y analítico relacional, con un diseño epidemiológico. En ella se realizaron 266 encuestas a personal de salud de la ciudad del área metropolitana de Buenos Aires. Los encuestadores fueron médicos y licenciados en nutrición cursantes del Posgrado en Actividad Física y Salud, perteneciente al Ministerio de Salud del Gobierno de la Ciudad de Buenos Aires, que recibieron capacitación específica para administrar un cuestionario. El instrumento utilizado fue el Cuestionario Mundial de Actividad Física (GPAQ), recordatorio de actividades de una semana habitual, propuesto por la Organización Mundial de la Salud (OMS) versión 2 en español. ${ }^{12}$ Este cuestionario recoge información acerca de diferentes características o dimensiones de la actividad física (intensidad, dominio). Consta de 16 preguntas y en este caso la pregunta referida a tiempo sedentario, fue desglosada por dominio (trabajo, transporte y recreación).

\section{Variables demográficas}

- Sexo: variable categórica, nominal y dicotómica: femenino y masculino.

- Edad: variable categórica, nominal y politómica, con los siguientes valores posibles: $<25,25$ a 34,35 a 44,45 a 54,55 a $64,>64$ años.

- Nivel de Actividad física (AF): variable categórica, y dicotómica: su valores son insuficiente y suficiente, que corresponde a actividad física moderada y vigorosa (AFMV). La AFMV engloba a los niveles moderado y vigoroso de actividad física. Esto permite resolver la dificultad que poseen este tipo de cuestionarios para diferenciar los niveles de intensidad. La recomendación de OMS sobre realización de actividad física, en relación a efectos protectores respecto a la salud, es de 150 minutos semanales, involucrando una intensidad moderada, durante la mayoría de los días de la semana. ${ }^{13}$ Se eligió por lo tanto ese valor como punto de corte.

- AFMV en el trabajo, transporte y tiempo recreativo, variables intervalares y discretas, cuyas unidades son los minutos.

Tiempo sedentario (TS) en el trabajo, transporte y tiempo recreativo, variables intervalares $y$ discretas, unidades en minutos. Se expresa en forma de valor semanal o promedio diario.

La circunferencia de la cintura (CC) se midió con una cinta métrica graduada en centímetros, con una precisión de $0,1 \mathrm{~cm}$, cinta flexible, pero no distensible, con el paciente de pie y en apnea espiratoria. Se midió el perímetro mínimo entre la cresta ilíaca y el borde costal inferior. La variable se categorizó y expresó como riesgo metabólico. La circunferencia de cintura se presenta como variable categórica y ordinal, y los valores posibles fueron: Bajo riesgo, Riesgo elevado, Riesgo muy elevado. Se pueden ver los valores de corte utilizados en la Tabla 1. 


\begin{tabular}{|l|c|c|c|}
\hline \multicolumn{4}{|c|}{ Tabla 1. Clasificación del Perímetro de Cintura en función de Riesgo Metabólico (cm) } \\
\hline & Bajo Riesgo & Riesgo elevado & Riesgo muy elevado \\
\hline Mujeres & $<80$ & $80-88$ & $>88$ \\
\hline Varones & $<94$ & $94-102$ & $>102$ \\
\hline Modificado de: Lean, 1995 (10)
\end{tabular}

Se calcularon valores de tendencia central, variabilidad y percentiles seleccionados para actividad física y tiempo sedentario. A los fines de estudiar la homogeneidad de varianzas en los distintos subgrupos se utilizó el test de Levene y luego se realizó ANOVA OneWay o la prueba no paramétrica $U$ de MannWhitney, según correspondiese, para mostrar diferencias significativas. Las diferencias entre grupos de edades para la AFMV en los dominios ocupacional, del transporte y del tiempo libre, considerados de manera independiente, fueron determinadas mediante la prueba $t$ de Student o la de Kruskal-Wallis, como alternativas paramétrica y no paramétrica, de acuerdo a los resultados del test de Levene. Estas últimas pruebas también se utilizaron para detectar diferencias entre los grupos de edades para el tiempo sedentario. Adicionalmente, se realizó una Regresión Logística Binomial integrando las variables sexo, edad y actividad física semanal. En todos los casos el nivel de significación fue establecido para $p<0.05$. El tratamiento estadístico fue realizado con el programa estadístico SPSS Statistics versión 20.0 (IBM Corp., Armor, New York).

Todos los encuestados fueron personas adultas y su participación fue voluntaria. El proyecto de investigación fue aprobado por el Comité Ético de Investigación de la Fundación Instituto Superior de Ciencias de la Salud: Código CISED 5/13 del 25/3/2013.

\section{RESULTADOS}

Se estudiaron 266 sujetos de ambos sexos (183 mujeres y 83 varones), entre los 24 y los 66 años de edad.

\begin{tabular}{|c|c|c|c|c|c|}
\hline & & $\begin{array}{c}\text { Trabajo } \\
\text { (minutos x día) }\end{array}$ & $\begin{array}{c}\text { Transporte } \\
\text { (minutos x día) }\end{array}$ & $\begin{array}{c}\text { Recreación } \\
\text { (minutos x día) }\end{array}$ & $\begin{array}{c}\text { Total } \\
\text { semanal }\end{array}$ \\
\hline \multicolumn{2}{|l|}{ Media } & 45.86 & 23.94 & 25.96 & 670.39 \\
\hline \multicolumn{2}{|c|}{ Desvío típico } & 77.03 & 34.98 & 30.42 & 706.45 \\
\hline \multicolumn{2}{|l|}{ Mínimo } & 0 & .00 & 0 & 0 \\
\hline \multicolumn{2}{|l|}{ Máximo } & 360 & 214.29 & 189 & 3480 \\
\hline \multirow{5}{*}{ Percentiles } & 18 & 0 & 0 & 0 & 145.3 \\
\hline & 19 & 0 & 0 & 0 & 150 \\
\hline & 25 & 0 & 0 & 0 & 201 \\
\hline & 50 & 0 & 12.86 & 17.14 & 440 \\
\hline & 75 & 68.57 & 30 & 34.82 & 900 \\
\hline
\end{tabular}


En la tabla 1, se presentan valores de tendencia central, variabilidad y percentiles seleccionados para AFMV en minutos por día en los dominios del trabajo, el transporte y el tiempo libre, además del tiempo total en minutos por semana. Se destaca una gran variabilidad en las variables, por ejemplo, la media semanal es de $670.39 \pm 706.45$ minutos (aproximadamente 11 horas y 10 minutos). Teniendo en cuenta que la recomendación de OMS sobre la actividad física moderada es de 150 minutos semanales ${ }^{13}$ se observa que el $18 \%$ de los sujetos estudiados reporta no alcanzar la recomendación, el resto la alcanza y supera.

Tabla 2. Tiempo Sedentario por dominio en minutos $(n=266)$

\begin{tabular}{|c|c|c|c|c|c|}
\hline \multicolumn{2}{|l|}{ 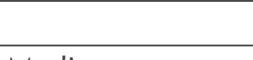 } & Trabajo & Transporte & Recreación & Total diario \\
\hline & 129.23 & 43.31 & 143.59 & 316.13 \\
\hline \multicolumn{2}{|c|}{ Desvío típico } & 127.84 & 47.45 & 83.32 & 143.62 \\
\hline \multicolumn{2}{|l|}{ Mínimo } & 0 & 0 & 0 & 0 \\
\hline \multicolumn{2}{|l|}{ Máximo } & 480 & 180 & 330 & 595 \\
\hline \multirow{3}{*}{ Percentiles } & 25 & 0 & 0 & 90 & 208.75 \\
\hline & 50 & 120 & 30 & 120 & 330 \\
\hline & 75 & 240 & 60 & 180 & 420 \\
\hline
\end{tabular}

En referencia al tiempo sedentario, en la tabla 2 se muestran valores de tendencia central, variabilidad y percentiles seleccionados, por día en diferentes dominios y para el total diario. Nuevamente se observa una variabilidad de resultados en todas las variables, con un valor de media de $316.13 \pm 143.62$ minutos para el total diario. El $50 \%$ de los sujetos refiere dedicar 330 minutos (5 horas y 30 minutos) por día a actividades sedentarias, y el $25 \%$ que reporta mayor tiempo diario dedicado a comportamientos sedentarios, destina más de 420 minutos (7 horas) por día a los mismos.

Tabla 3. Actividad física Moderada y Vigorosa (AFMV) y tiempo sedentario (TS) según sexo Medias $(X)$ y desvíos estándar (S)

\begin{tabular}{|l|c|c|c|c|c|c|c|c|c|}
\hline Sexo & $\begin{array}{c}\text { AFMV } \\
\text { Trabajo }\end{array}$ & $\begin{array}{c}\text { AFMV } \\
\text { transporte }\end{array}$ & $\begin{array}{c}\text { AFMV } \\
\text { recreación }\end{array}$ & $\begin{array}{c}\text { AFMV } \\
\text { semana }\end{array}$ & $\begin{array}{c}\text { TS } \\
\text { trabajo }\end{array}$ & $\begin{array}{c}\text { TS } \\
\text { transporte }\end{array}$ & $\begin{array}{c}\text { TS } \\
\text { recreación }\end{array}$ & $\begin{array}{c}\text { TS } \\
\text { transporte }\end{array}$ \\
\hline M & $\mathrm{X}$ & 44.15 & 21.83 & 27.38 & 653.54 & 137.17 & 34.28 & 157.47 & 34.28 \\
\cline { 2 - 11 } 83 & $\mathrm{~S}$ & 84.22 & 36.72 & 28.23 & 701.81 & 131.8 & 43.18 & 85.67 & 43.18 \\
\hline $\mathrm{F}$ & $\mathrm{X}$ & 46.64 & 24.9 & 25.32 & 678 & 125.63 & $47.4 *$ & 137.3 & $47.4 *$ \\
\cline { 2 - 10 } 183 & S & 73.77 & 34.32 & 31.42 & 710.33 & 126.61 & 48.82 & 81.7 & 48.82 \\
\hline
\end{tabular}

En la tabla 3 se aprecian las medias y desvíos estándar, según sexo, para AFMV y TS. Luego de verificar si hubo homogeneidad de varianzas en los distintos subgrupos, a través del test de Levene, se comprobó mediante el t-Test para grupos independientes, que solo hubo diferencias significativas en el Tiempo Sedentario en el transporte $(t=2.1 ; p=0.036)$. 


\begin{tabular}{|c|c|c|c|c|c|c|c|c|c|}
\hline \multirow[b]{2}{*}{$\begin{array}{l}\text { Edad } \\
\text { Rango n(\%) }\end{array}$} & \multirow[b]{2}{*}{$\mathrm{X} / \mathrm{S}$} & \multicolumn{8}{|c|}{ Medias $(X)$ y desvíos estándar $(S)$} \\
\hline & & $\begin{array}{l}\text { AFMV } \\
\text { trabajo }\end{array}$ & $\begin{array}{c}\text { AFMV } \\
\text { transporte* }\end{array}$ & \begin{tabular}{|c|} 
AFMV \\
recreación
\end{tabular} & $\begin{array}{c}\text { AFMV } \\
\text { semanal }\end{array}$ & $\begin{array}{c}\mathrm{TS} \\
\text { trabajo }\end{array}$ & \begin{tabular}{|c|}
$\mathrm{TS}$ \\
transporte
\end{tabular} & \begin{tabular}{|c|} 
TS \\
recreación
\end{tabular} & $\begin{array}{l}\text { TS } \\
\text { día }\end{array}$ \\
\hline \multirow{2}{*}{$\begin{array}{l}<25 \\
8(3)\end{array}$} & $x$ & 13.93 & 15.71 & 31.79 & 430 & 67.5 & 67.5 & 165 & 300 \\
\hline & $\mathrm{S}$ & 23.33 & 19.81 & 36.95 & 315.73 & 57.26 & 72.65 & 42.43 & 70.1 \\
\hline \multirow{2}{*}{$\begin{array}{l}25 / 34 \\
92(34.6)\end{array}$} & $X$ & 52.51 & 23.68 & 28.55 & 733.15 & 115.76 & 41.68 & 145.22 & 302.66 \\
\hline & $\mathrm{S}$ & 83.04 & 37 & 30.15 & 756.62 & 115 & 46.95 & 78.64 & 147.62 \\
\hline \multirow{2}{*}{$\begin{array}{l}35 / 44 \\
89(33.5)\end{array}$} & $X$ & 34.49 & 25.42 & 23.26 & 582.19 & 130.28 & 47.36 & 133.6 & 311.24 \\
\hline & $\mathrm{S}$ & 63.38 & 29.93 & 29.42 & 553.91 & 136.3 & 46.07 & 86.84 & 141.85 \\
\hline \multirow{2}{*}{$\begin{array}{l}45 / 54 \\
48(18)\end{array}$} & $X$ & 59.24 & 31.58 & 24.23 & 805.31 & 145.21 & 45.31 & 156.25 & 346.77 \\
\hline & $\mathrm{S}$ & 86.68 & 45.31 & 25.72 & 801.52 & 120.43 & 51.84 & 86.14 & 129.28 \\
\hline \multirow{2}{*}{$\begin{array}{l}55 / 64 \\
26(9.8)\end{array}$} & $X$ & 48.41 & 7.75 & 29.14 & 597.08 & 161.54 & 26.73 & 143.65 & 331.92 \\
\hline & $\mathrm{S}$ & 91.9 & 15.73 & 41.64 & 861.67 & 163.68 & 33.55 & 92.97 & 168.43 \\
\hline \multirow{2}{*}{$\begin{array}{l}>64 \\
3(1.1)\end{array}$} & $x$ & 28.57 & 28.57 & 11.43 & 480 & 140 & 20 & 130 & 290 \\
\hline & $\mathrm{S}$ & 49.49 & 49.49 & 9.9 & 729.93 & 124.9 & 34.64 & 86.6 & 233.02 \\
\hline
\end{tabular}

Se muestran, en la tabla 4, las medias y desvíos estándar de AFMV y TS, de acuerdo a categorías de edad. A continuación de realizar, en los distintos subgrupos, el test de Levene, se comprobó, mediante la prueba de KruskalWallis, que solo hubo diferencias significativas en la AFMV en el transporte $\left(\chi^{2}=13.72 ; p=0.014\right)$. En las variables que presentan diferencias significativas se pueden observar los valores de las medias para las distintas categorías de edad, para constatar entre que grupos se encuentran las diferencias.

Respecto al riesgo metabólico por el perímetro de cintura, del total de los sujetos estudiados, el $65.8 \%$ (175) presentan un riesgo bajo, 18.4\% (49 casos), un riesgo elevado, y $15.8 \%$ (42 casos) tienen un riesgo muy elevado. Se pueden entonces relacionar las categorías de Riesgo Metabólico con Actividad física como se presenta en tabla 5.

Tabla 5. Actividad Física Moderada y Vigorosa (AFMV) y Tiempo Sedentario (TS) según categorías de riesgo metabólico (RM) - Medias (X) y desvíos estándar (S)

\begin{tabular}{|l|c|c|c|c|c|c|c|c|c|}
\hline RM & X/S & $\begin{array}{c}\text { AFMV } \\
\text { trabajo }\end{array}$ & $\begin{array}{c}\text { AFMV } \\
\text { transporte }\end{array}$ & $\begin{array}{c}\text { AFMV } \\
\text { recreación* }\end{array}$ & $\begin{array}{c}\text { AFMV } \\
\text { semanal }\end{array}$ & $\begin{array}{c}\text { TS } \\
\text { trabajo }\end{array}$ & $\begin{array}{c}\text { TS } \\
\text { transporte }\end{array}$ & $\begin{array}{c}\text { TS } \\
\text { recreación }\end{array}$ & $\begin{array}{c}\text { TS } \\
\text { día* }\end{array}$ \\
\hline BAJO & $\mathrm{X}$ & 47.73 & 25.15 & 28.77 & 711.54 & 120.97 & 44.2 & 136.94 & 302.11 \\
\cline { 2 - 10 } n:175 & $\mathrm{S}$ & 77.54 & 37.22 & 30.13 & 727.86 & 121.26 & 47.49 & 76.49 & 144.7 \\
\hline $\begin{array}{l}\text { ALTO } \\
\text { n:49 }\end{array}$ & $\mathrm{X}$ & 41.68 & 17.35 & 20.58 & 557.24 & 119.08 & 48.67 & 157.96 & 325.71 \\
\cline { 2 - 10 } & $\mathrm{S}$ & 67.27 & 25.32 & 32.6 & 586.73 & 129.05 & 57.02 & 91.14 & 147.86 \\
\hline $\begin{array}{l}\text { Muy ALTO } \\
\text { n:42 }\end{array}$ & $\mathrm{X}$ & 42.99 & 26.6 & 20.54 & 630.95 & 175.48 & 33.33 & 154.52 & 363.33 \\
\cline { 2 - 9 } & $\mathrm{S}$ & 86.58 & 34.82 & 28.04 & 741.77 & 145.35 & 32.15 & 98.78 & 125.02 \\
\hline
\end{tabular}


Se realizaron contrastes por categorías de riesgo metabólico por perímetro de cintura, a través de la prueba de Kruskal-Wallis, observándose diferencias significativas en AFMV diaria $\left(\chi^{2}=8.57 ; p=0.014\right)$ y el Tiempo Sedentario diario $\left(\chi^{2}=6.14 ; p=0.046\right)$.

Se estudió la asociación entre el nivel de actividad física y el riesgo metabólico por perímetro de cintura. El valor de Tau-c de Kendall obtenido fue de -0.097 , indicando que la relación, como era esperable, es negativa (a mayor nivel de actividad física, menor riesgo), pero considerando que los valores de la medida de asociación considerada pueden estar entre -1 y +1 , el valor hallado muestra una asociación muy débil y no significativa entre ambas variables. La relación entre el tiempo sedentario y el riesgo metabólico (considerado como variable dependiente) se calculó a través del coeficiente Eta, cuyo valor fue de 0.504. También aquí se observa una asociación débil entre ambas variables, ya que el valor de Eta2 de 0.254 indica que el tiempo total sedentario explica sólo un $25.64 \%$ de la variabilidad del riesgo metabólico.

Para conocer si el sexo, la edad, el nivel de actividad física, la actividad física moderada a vigorosa semanal promedio en minutos $\mathrm{y} / \mathrm{o}$ el tiempo sedentario diario promedio en minutos, eran buenos predictores de la variable no riesgo/ riesgo metabólico por perímetro de cintura, se realizó una Regresión Logística Binomial. Los valores de $\chi^{2}$ para el modelo, en un único paso posible, mostraron una significación menor a 0,05, que indicaba que una sola variable independiente, la edad, explicaba la dependiente, el riesgo metabólico, clasificando correctamente el $66.2 \%$ de los casos, valor superior al 50\% requerido. Surge entonces que la edad es la única variable independiente que explica el riesgo metabólico por perímetro de cintura.

\section{DISCUSIÓN}

Existen varios métodos para el diagnóstico de obesidad y los métodos antropométricos, aunque sencillos, parecen ser tan efectivos como aquellos más sofisticados como la tomografía computarizada, la absorciometría de rayos $\mathrm{X}$ de energía dual (DEXA) y la resonancia magnética. ${ }^{15}$ Los indicadores antropométricos más utilizados son el índice de masa corporal (IMC), la circunferencia de la cintura, el índice cintura-cadera (ICC) y la sumatoria de pliegues cutáneos. En la práctica profesional diaria es deseable un método simple, de bajo costo y de fácil interpretación que pueda ser utilizado en promoción de la salud y prevención de factores de riesgo cardiovascular. La circunferencia de la cintura es una variable antropométrica que posee una buena correlación con el tejido adiposo visceral comparando con pruebas de diagnóstico por imagen como la resonancia magnética y la tomografía computada. Los puntos de corte utilizados para la clasificación de la circunferencia de cintura fueron definidos por Lean ${ }^{14}$ en un estudio transversal realizado en Glasgow en 1995. En ese entonces se identificaron los siguientes puntos de corte: una circunferencia $\geq 94 \mathrm{~cm}$ para hombres $y \geq 80$ $\mathrm{cm}$ para mujeres que identificó sujetos con un $I M C \geq 25 \mathrm{~kg} / \mathrm{m}$ con una sensibilidad del $>96 \%$ y una especificidad $>97.5 \%$. Una $C C \geq 102 \mathrm{~cm}$ para hombres $\mathrm{o} \geq 88 \mathrm{~cm}$ para mujeres identificó sujetos con un $I M C \geq 30$ con una sensibilidad $>96 \%$ y una especificidad $>98 \%$. Para promoción de la salud, estos puntos de corte pueden ser utilizados sugiriendo a los hombres con $\mathrm{CC} \geq 94 \mathrm{~cm}$ y a mujeres con $\mathrm{CC} \geq 80 \mathrm{~cm}$ que no deberían incrementar su peso. Por otro lado, a los hombres con $\mathrm{CC} \geq 102 \mathrm{~cm}$ y a las mujeres con $\mathrm{CC} \geq 88 \mathrm{~cm}$ se les recomienda disminuir de peso. Los puntos de corte específicos para cada sexo se pueden utilizar para identificar un mayor riesgo asociado a la grasa abdominal en adultos con un IMC en el rango de 25 a $34.9 \mathrm{~kg} / \mathrm{m} 2$. Un aumento en la circunferencia de la cintura también puede estar asociado a un mayor riesgo en personas de peso normal. ${ }^{12}$ Los puntos de corte por lo general se pueden aplicar a todos los grupos étnicos o raciales adultos. Pero si una persona es de baja estatura, mide menos de $150 \mathrm{~cm}$, o tiene un IMC por encima de $34.9 \mathrm{~g}$ $\mathrm{m} 2$, los puntos de corte para población general deberían ser evitados. ${ }^{16}$

En este trabajo se integraron los niveles moderado y vigoroso de actividad física ya que ambos niveles impactan a nivel salud. La recomendación de OMS sobre realización de 
actividad física, en relación a efectos protectores respecto a la salud, es de 150 minutos semanales de actividad física de intensidad moderada, durante la mayoría de los días de la semana. ${ }^{13}$ Se observa en la Tabla 2 que el $18 \%$ de los sujetos estudiados reporta no alcanzar la recomendación, mientras que el $82 \%$ la alcanza y la supera. En lo que hace a tiempo sedentario, el $50 \%$ de los sujetos refiere dedicarle 330 minutos diarios a este comportamiento, o sea unas 5.5 horas por día. Es más, el 25\% que reporta mayores valores, le destina más de 420 minutos, o sea 7 horas. Se debe recordar que el tiempo sedentario implica un riesgo para la salud, que es independiente de los niveles de actividad física alcanzados, y que, incluso, puede contrarrestar sus beneficios. Por lo tanto la actividad y el comportamiento sedentario son distintos factores de riesgo que pueden afectar de forma independiente a la salud. ${ }^{17}$ La conducta sedentaria se caracteriza por actividades de bajo gasto energético, 1.0-1.5 Mets, en el tiempo de vigilia, como sentarse o acostarse. ${ }^{18}$ La conducta sedentaria ha sido asociada a un mayor riesgo de enfermedades crónicas, incluyendo el síndrome metabólico. ${ }^{19}$

George y colaboradore ${ }^{20}$ realizaron un trabajo sobre una muestra de 63.048 varones australianos en el estudio The 45 and Up Study, un estudio de cohorte longitudinal sobre envejecimiento saludable. Estudiaron el tiempo sedentario y los agruparon en quienes reportaban estar sentados $<4$ horas/día, 4 a $<6,6$ a $<8$ y $\geq 8$ horas. Los que pasaban 4 o más horas sentados al día fueron significativamente más propensos a reportar haber tenido alguna enfermedad crónica (con una razón de probabilidad ajustada (AOR) 1,06, IC del 95\%: 1.00 - 1.12, $\mathrm{p}=0.050$; AOR 1,10; IC del 95\%: 1,03 a 1,16; $p=0.003$; AOR 1.09, IC $95 \% 1,03-1,15, p=0.002$, respectivamente) y especialmente diabetes (AOR 1.15; IC del 95\%: 1.03 hasta 1.28, $\mathrm{p}=0.016$; AOR 1.21; IC del 95\%: 1.09 a $1.33, p<0,001$, respectivamente). Los autores sugieren que los mayores volúmenes de tiempo sentados se asocian significativamente con diabetes y las enfermedades crónicas en general, independiente de la actividad física. ${ }^{20}$ En el presente trabajo, el $50 \%$ de la muestra presentó mayores valores de tiempo sedentario.

El trabajo de Henson realizado en el Reino
Unido $^{21}$ sobre adultos con alto riesgo de diabetes mellitus tipo 2, muestra que el tiempo sedentario se asocia fuertemente y de manera adversa con la salud cardiometabólica y puede ser un indicador más importante de salud que la misma actividad física. Este estudio examinó las asociaciones entre el tiempo sedentario y la actividad física utilizando acelerómetros ActiGraph GT3X y marcadores de salud cardiometabólicos en 878 participantes con factores de riesgo conocidos para diabetes mellitus tipo 2. Se encontró asociaciones lineales perjudiciales de tiempo sedentario con glucosa plasmática $(\beta=0.220$, $p<0,001)$, triglicéridos $(\beta=0.206, \quad p=0.001)$ y HDL-colesterol $(\beta=-0.123, \quad p=0.029)$. La actividad física fue significativa e inversamente asociada con medidas de adiposidad. ${ }^{21}$ Concluyen los autores que el tiempo sedentario se puede relacionar de modo concluyente con la salud cardiometabólica.

Los estudios de tiempo sedentario muestran asociaciones perjudiciales con la obesidad y otros precursores metabólicos de enfermedades crónicas como diabetes tipo 2, enfermedades cardiovasculares y cáncer de mama y de colon. $^{22}$ El personal de salud estudiado y la población en general pasan mucho tiempo sedente, trabajando frente a una pantalla, en el transporte y en el tiempo de ocio. En definitiva, aun cuando se cumplan las pautas de actividad física, muchos están sentados por períodos prolongados, lo que puede comprometer su salud. Independientemente de si además, sean activos físicamente.

\section{CONCLUSIONES}

Al estudiar a profesionales de la salud en la ciudad de Buenos Aires, se observó que solo el $18 \%$ no cumple con las recomendaciones de actividad física. Sin embargo el 50\% de los sujetos estudiados reporta más de 5.5 horas diarias de conducta sedentaria, y lo que es aún más preocupante, el $\sim 25 \%$ refiere 7 o más horas. A pesar de las limitaciones del método (medidas subjetivas, que sobrestiman la actividad física y subestiman conducta sedentaria) se pudo determinar que los sujetos con menor riesgo metabólico posen mayores niveles de actividad física, principalmente de 
tipo recreativo, y un menor tiempo semanal de conducta sedentaria. Por último, el incremento de la edad fue relacionada con un aumento del riesgo metabólico. Esto coincide con las publicaciones internacionales y muestra un área de intervención posible, aumentando los niveles de actividad física e introduciendo pausas activas para disminuir los tiempos prolongados de conducta sedentaria.

\section{REFERENCIAS BIBLIOGRÁFICAS}

1. Stamatelopoulos KS, Lekakis JP, Vamvakou G, Katsichti P, Protogerou A, Revela I, et al. The relative impact of different of adiposity on markers of early atherosclerosis. Int J Cardiol. 2007; 119(2):139-46.

2. de la Fuente Crespo RV, Carballo Martínez RG, Fernández-Britto Rodríguez JE, Sirsi Guilarte Díaz S, Albert Cabrera MJ. Circunferencia de la cintura con sobrepeso e hipertensión arterial en adultos. Revista Habanera de Ciencias Médicas. 2012; 11(S5): 650-64.

3. Hubert HB, Feinleib $M, M$ McNamara, Castelli W. Obesity as an independent risk factor for cardiovascular disease: A 26-year follow-up of participants in the Framingham Heart Study. Circulation. 1983; 67(5): 96876.

4. Peixoto MRP, Benício MHD, Latorre MRDO, Jardim PCBV. Circunferência da cintura e índice de massa corporal como preditores da hipertensão arterial. Arq Bras Cardiol. 2006; 87 (4): 462-70.

5. Thornton JS, Frémont $\mathrm{P}$, Khan $\mathrm{K}$, Poirieer $\mathrm{P}$, Fowles J, Wells GD, et al. Physical activity prescription: a critical opportunity to address a modifiable risk factor for the prevention and management of chronic disease: a position statement by the Canadian Academy of Sport and Exercise Medicine. Br J Sports Med Published Online First: June 22, 2016 as 10.1136/bjsports-2016-096291

6. Reilly M, Ayala GX, Elder JP, Patrick K. Physician communication and physical activity among latinas. Journal of Physical Activity and Health. 2013; 10: 602-6.

7. Orrow G, Kinmonth AL,Sanderson S, Sutton S. Effectiveness of physical activity promotion based in primary care: systematic review and meta-analysis of randomised controlled trials. BMJ. 2012 March 26; 344:e1389.

8. Sjöling M, Lundberg K, Englund E, Westman A, Jong MC. Effectiveness of motivational interviewing and physical activity on prescription on leisure exercise time in subjects suffering from mild to moderate hipertension. BMC Research Notes, 2011; 4:352. doi:10.1186/1756-0500-4-352

9. Grandes G, Sanchez A, Sanchez-Pinilla RO, Torcal J, Montoya I, Lizarraga K, et al. Effectiveness of physical activity advice and prescription by physicians in routine primary care: a cluster randomized trial. Arch Intern Med. 2009 Apr 13;169(7):694-701. doi: 10.1001/archinternmed.2009.23.

10. Barnes PM, Schoenborn ChA. Trends in adults receiving a recommendation for exercise or other physical activity from a physician or other health professional. National Center for Health Statistics (NCHS) Data Brief. 2012; 86: 1-8.

11. Frank E, Tong E, Lobelo F, Carrera J, Duperly J. Physical activity levels and counselling practices of U.S. Medical students. Medicine and Science in Sports and Exercise. 2008; 40: 413-21.

12. Organización Mundial de la Salud. El método STEPwise de la OMS para la vigilancia de los factores de riesgo de las enfermedades crónicas; 2007. [sitio en internet] [Acceso 5/3/2013] Disponible en: http://www.who. int/chp/steps/instrument/es/index.html

13. Organización Mundial de la Salud. Recomendaciones mundiales sobre actividad física para la salud. Ginebra, Suiza: OMS; 2010.

14. Lean ME, Han TS, Morrison CE. Waist circumference as a measure for indicating 
need for weight management. BMJ. 1995 Jul 15; 311(6998):158-61.

15. Kuper H, Taylor A, Krishna KV, Ben-ShlomoY, Gupta R, Kulkarni B, Prabhakaran D, y otros. Is vulnerability to cardiometabolic disease in Indians mediated by abdominal adiposity or higher body adiposity. BMC Public Health . 2014;14:1239. doi:10.1186/1471-2458-141239

16. Clinical guidelines on the identification, evaluation, and treatment of overweight and obesity in adults. Guidelines on overweight and obesity. National Heart, Lung, and Blood Institute; 1998. [sitio en internet] [Acceso 2/12/2014] Disponible en: http://www. nhlbi.nih.gov/health-pro/guidelines/current/ obesity-guidelines/e_textbook/txgd/4142. htm

17. Healy GN, Wijndaele K, Dunstan DW, Shaw JE, Salmon J, Zimmet PZ, et al. Objectively measured sedentary time, physical activity, and metabolic risk. Diabetes Care. 2008; 31:369-71.

18. Pate RR, O'Neill JR, Lobelo F. The evolving definition of "sedentary". Exerc Sport Sci Rev. 2008; 36:173-8.

19. Dunstan DW, Salmon J, Owen N, Armstrong T, Zimmet PZ, Welborn T, et al. Associations of TV viewing and physical activity with the metabolic syndrome in Australian adults. Diabetologia. 2005; 48:2254-61.

20. George ES, Rosenkranz RR, Kolt GS. Chronic disease and sitting time in middle-aged Australian males: findings from the 45 and Up Study. International Journal of Behavioral Nutrition and Physical Activity. 2013; 10:20.

21. Henson J, Yates T, Biddle SJ, Edwardson CL, Khunti K, Wilmot EG, et al. Associations of objectively measured sedentary behaviour and physical activity with markers of cardiometabolic health. Diabetologia. 2013 May; 56(5):1012-20. doi: 10.1007/s00125013-2845-9

22. Owen N, Healy GN, Matthews CE, Dunstan DW. Too much sitting: The populationhealth science of sedentary behavior. Exerc Sport Sci Rev. 2010 July; 38(3): 105-13. 